\title{
Duration of antiretroviral regimens in treatment- experienced patients in clinical practice
}

\author{
Vicente Escudero Vilaplana*, Sergio Plata Paniagua, Nicolas Trovato Lopez, Isabel Castillo Romera, \\ Arantza Ais Larisgoitia, Jose Maria Bellon Cano, Maria Sanjurjo Saez \\ From $16^{\text {th }}$ International Symposium on HIV and Emerging Infectious Diseases \\ Marseille, France. 24-26 March 2010
}

\section{Background}

Antiretroviral regimens (ART) with few changes due to side effects or treatment failure are preferred. Therefore, duration of treatment and persistence (defined as continuing therapy or not) are useful measures of success of ART. We studied duration of ART in treatment-experienced patients (TEP) and analyzed how a history of non-adherence affects it.

\section{Methods}

In September 2009, we conducted a retrospective, observational study of adult TEP whose ART was switched between 01/05/2008 and 30/04/2009. We used pharmacy records to select all patients who switched an ART containing darunavir, raltegravir, maraviroc, and/or etravirine and patients who had switched regimens not containing these drugs (1:1) on the same day. The primary endpoint was duration of treatment from inclusion until the last refill, or until the first refill of a new regimen. Patients were classified as non-adherent if they had collected less than $90 \%$ of the doses needed during the year before inclusion. Control variables were viral load (VL), CD4 count at inclusion, and time since first ART regimen (tART). Persistence was estimated using Kaplan-Meier plots. Groups were compared using the log-rank test and a Cox regression model was adjusted for control variables.

\section{Results}

We included 146 patients (66.4\% men); mean age 45.4 years. Baseline clinical characteristics (median [IQR]) were $\mathrm{VL}=50(50-7331)$ copies $/ \mathrm{mL}, \mathrm{CD} 4$ count $=345$ (184-540) cells $/ \mu \mathrm{L}$, tART $=10.0(4.2-10.7)$ years.
Etravirine, maraviroc, and/or raltegravir were administered to $45.9 \%$ of patients, and efavirenz to $21.9 \%$.

Persistence $(95 \% \mathrm{CI})$ at 6 and 12 months was $77.9 \%$ (70.9\%-84.9\%) and 65.9\% (55.5\%-76.5\%), respectively. Mean duration of treatment was 342 (15) days. Nonadherence was observed in $29.3 \%$ (21.2\%-37.3\%). Persistence at 12 months for adherent and non-adherent patients was $71.7 \%(58.1 \%-85.3 \%)$ vs $59.0 \%$ (32.2\%$84.8 \%)$, respectively $(\mathrm{p}=0.342)$. There were no differences in persistence adjusted for control variables (HR, 0.774 [0.343-1.750]; $\mathrm{p}=0.539$ ).

After a median 281 days of follow-up, 24.7\% (17.731.6) had stopped or changed ART because of toxicity $(52.8 \%)$, treatment failure $(19.4 \%)$, or simplification (8.3\%).

\section{Discussion}

Our results for persistence are similar to published data for naïve patients and toxicity is also the main reason for switching treatment. A history of non-adherence has less effect on persistence than expected.

Published: 11 May 2010

doi:10.1186/1742-4690-7-S1-P166

Cite this article as: Vilaplana et al:: Duration of antiretroviral regimens in treatment-experienced patients in clinical practice. Retrovirology 2010 7(Suppl 1):P166.

* Correspondence: vescudero.hgugm@salud.madrid.org

Hospital General Universitario Gregorio Marañon, Madrid, Spain 